\title{
El Modelo de Escuela Rural ¿Es un Modelo Transferible a Otro Tipo de Escuela?
}

\author{
Pilar Abós Olivares \\ 'Universidad de Zaragoza, Teruel - España
}

RESUMEN - El Modelo de Escuela Rural $i$ Es un Modelo Transferible a Otro Tipo de Escuela? El trabajo presenta el diseño y los primeros datos de la investigación realizada en el marco de un Proyecto $\mathrm{I}+\mathrm{D}+\mathrm{I}^{1}$ del Ministerio de Ciencia e Innovación español y en el que han participado diferentes universidades europeas y latinoamericanas ${ }^{2}$ y que pretendía abrir vías de reflexión y diseñar propuestas sobre la adquisición de competencias en la escuela rural y cómo estas competencias trabajadas en el marco de una metodología participativa-activa, con una gran atención a la diversidad cultural y también social, puede tener componentes didácticos y organizativos transferibles a las escuelas ordinarias.

Palabras-clave: Escuela Rural. Didáctica Multigrado. Metodología Activa.

ABSTRACT - The Rural School Model - Is that a Model Transferable to Other Schools? The paper presents the design and preliminary data from a research conducted in the framework of an I + D + I project of the Spanish Ministry of Science and Innovation in which various European and Latin American universities have participated. It intended to open up avenues for reflection and to design proposals for the acquisition of skills in rural schools and how these skills, developed according to a participatory-active methodology framework, with great attention to cultural and social diversity, can have educational and organizational components transferable to regular schools.

Keywords: Rural School. Multigrade Teaching. Active Methodology.

Educação \& Realidade, Porto Alegre, v. 40, n. 3, p. 667-684, jul./sept. 2015. 


\section{Introducción}

La pregunta que ilustra el título de este artículo requiere unas breves líneas que nos ayuden a comprender las dificultades y posibilidades que nos ofrece la escuela rural del siglo XXI, fruto de las importantes transformaciones producidas en los contextos rurales que nos conducen hacia una nueva identidad de la escuela rural en una sociedad con características complejas y diversas. Además, las dificultades que ha tenido y tiene la educación en el medio rural son consecuencia de hondas disfunciones que no se circunscriben al ámbito estrictamente pedagógico sino que abarcan aspectos demográficos, ideológicos y económicos, entre otros. Por este motivo para poder plantear las posibles aportaciones de la escuela rural al logro de una escuela de calidad consideramos necesario realizar algunas precisiones básicas que nos sitúen en el marco no sólo de las políticas educativas, sino de otras de carácter más general y que incluyen aspectos sociales y económicos.

Hablar de escuela en el medio rural implica una forzosa referencia a un escenario que refleja una manera de entender lo social, lo económico, la equidad y la redistribución de la riqueza (Bernat, 2009), y que se concreta en opciones políticas, la mayoría de las cuales se han olvidado de este tipo de escuela, aplicando estrategias y líneas de actuación iguales para todos los contextos (Martínez; Bustos, 2011). Este hecho es más significativo en el momento actual en el que prima la introducción de la educación en el mercado en el marco de un pensamiento neoliberal y neoconservador que no distingue territorios (Aróstegui; Martínez, 2008), de modo que la escuela rural está condicionada por una forma de entender la sociedad y la educación que desarrolla un modelo de racionalización centrado en el control, en la consecución del logro y en la competitividad y que plantean un modelo de escuela estandarizada en el que se prima la homogeneidad, potenciada a su vez por la tendencia a la superación de la espacialidad gracias a la mejora de las comunicaciones, tanto territoriales como virtuales (Sanz, 2009). Sin embargo, y aunque los cambios en educación no se produzcan por el mero hecho de legislar (Bernat, 2003), las características propias de la escuela rural exigen un tratamiento específico y diferenciado a partir de una organización y estructura acordes con las necesidades de cada contexto (Lorenzo; Santos, 2004).

El discurso y el razonamiento de "oposición" a la escuela urbana (Popkewitz, 1998) no sirve de modo exclusivo para apoyar ese tratamiento, sino la consideración de la diversidad y la heterogeneidad como característica específica, compartida con la urbana, y convertida en un reto para la mejora. Diversidad que no solo está presente dentro de la escuela sino que se identifica en la existencia de condiciones socioculturales heterogéneas y en la diversidad de contextos y territorios rurales (Rojas, 2006), así como en la reactivación de mecanismos de redefinición identitaria y de búsqueda de distintividad (Sanz, 2009).

668 Educação \& Realidade, Porto Alegre, v. 40, n. 3, p. 667-684, jul./sept. 2015. 
Ante esta situación la escuela rural puede situarse de modo automático en el sistema, conformándose y resignándose, o adoptar una actitud crítica, interpretando que su primera función es la de formar personas libres que tomen conciencia de su situación y que luchen por convertirse en protagonistas en la historia de su emancipación (Bernat, 2009).

En esta última postura se enmarca el proyecto que presentamos, partiendo de la convicción de que, cualquiera que sea el contexto en el que se ubique la escuela, esta debe atender a las diferencias individuales estimulando un aprendizaje autónomo y colaborativo y potenciando aquellas estrategias didácticas que tengan en cuenta la diversidad de capacidades, intereses y contextos con el último objetivo de un aprendizaje significativo.

\section{La Escuela Rural: respuestas imaginativas e innovadoras}

Cuando nos acercamos a lo que protagonizan muchos de los actores de la escuela rural incluso en contextos sociales y territoriales diferentes, visualizamos escenas con grandes posibilidades de proyección no solo didáctica sino también social, de creación de alternativas una escuela hegemónica uniformizadora y de potenciación de políticas sociales que defiendan valores de justicia y de solidaridad. Esta visión retoma la de una escuela con capacidad de descubrir, de experimentar y de protagonizar desde el territorio que la contiene y en la que alcanza su verdadera significación.

La escuela rural se convierte en un espacio para la innovación y la mejora exprimiendo aquellas potencialidades que, aunque en muchas ocasiones hayan sido consideradas como deficiencias, posibilitan el desarrollo de prácticas pedagógicas aplicables a cualquier situación educativa (Santos, 2011). Señalaremos algunas de ellas:

\section{La Heterogeneidad y Diversidad Posibilitan la Inclusión}

Stainback; Stainback (2007) consideran la educación inclusiva como un modelo de igualdad al considerar a todos los niños y niñas como únicos e irrepetibles y promover el desarrollo de sus múltiples potencialidades. De este modo, no sólo es un modelo que fomenta una educación de calidad entendida como educación integral que garantiza un aprendizaje experiencial y contextualizado (Miller, 2006), sino que garantiza el pleno ejercicio del derecho a la educación de todos los ciudadanos. En relación con esta idea nos encontramos que en la escuela rural los grupos están formados por alumnos de edades, intereses, capacidades y expectativas diferentes (Boix, 2009), lo que la convierte en un espacio educativo, que al estar constituido de forma heterogénea, en el que las prácticas inclusivas se convierten en la única forma de 
conseguir un aprendizaje significativo en todos los alumnos, valorando la diversidad y ofreciendo a todos y cada uno diferentes oportunidades de aprendizaje.

\section{El Medio Posibilita un Aprendizaje Experiencial}

La escuela rural se encuentra en espacios vinculados estrechamente al territorio sobre el que está construida y el territorio se caracteriza justamente porque tiene identidad propia (Bustos, 2009). Esta identidad que en ocasiones ha sido considerada como inferior debe, sin embargo, servir para convertir el medio rural en un medio de futuro, no de marginación y a la escuela que en él se ubica en un instrumento que la defienda y garantice, asumiendo el papel de revalorizar el saber local frente al proceso homogeneizador y hegemónico (Sepúlveda; Gallardo, 2011). Si aceptamos las premisas anteriores el currículo deberá tener en cuenta las necesidades de la población y sus marcos socioculturales, y permitirá un aprendizaje que, partiendo de los conocimientos previos, acerque al alumno desde sus propias vivencias hasta conceptos abstractos y generalizables que le conduzcan a una igualdad de resultados partiendo de condiciones diferentes.

La capacidad de aprender de forma autónoma, de aprender a aprender exige la habilidad para aprovechar nuestra interacción con el medio para construir conocimiento, de manera que cuando el alumno se enfrenta a situaciones reales se consolida un aprendizaje significativo y contextualizado, transferible y funcional. La experiencia como promotora de conocimiento, tal y como indicaba Dewey, nos conduce a afirmar que los individuos aprenden cuando encuentran significado en su interacción con el medio, y el medio en el que se encuentra la escuela rural es idóneo para potenciarla. El arraigo con el territorio permitirá el logro de aprendizajes contextualizados con la utilización de importantes recursos personales y materiales y la implicación de toda la comunidad educativa, aprovechando todo el potencial social que proporciona y creado personas autónomas, capaces de construir y reconstruir su entorno.

\section{El Aprendizaje Cooperativo como Apoyo de la Individualidad}

El binomio enseñanza-aprendizaje es inseparable porque enseñanza y aprendizaje son actividades compartidas en las que docentes, alumnos y saberes establecen continuos intercambios que permiten construir aprendizajes. El compromiso, la colaboración, el respeto y la tolerancia se convierten en instrumentos fundamentales para la transmisión de la información y el logro de la formación, de manera que el aprendizaje deja de ser una actividad estrictamente individual, convirtiendo el saber en saber con, al obtenerse a través de la cooperación y el intercambio que potencian el enriquecimiento mutuo.

670 Educação \& Realidade, Porto Alegre, v. 40, n. 3, p. 667-684, jul./sept. 2015. 
El conflicto cognitivo que pone en relación lo que sabemos con los nuevos saberes y que está en la base de la construcción del conocimiento se convierte en un aula diversa y heterogénea en conflicto sociocognitivo, permitiendo tanto el aprendizaje de habilidades sociales como de la capacidad de autonomía de aprender a aprender.

Por todo ello la escuela rural es un entorno y espacio idóneo para el aprendizaje y la renovación pedagógica y permite potenciar la regeneración del conjunto del sistema educativo en el actual contexto social y cultural permitiendo:

- Un desarrollo global del alumno en el marco de una escuela pluralista

- El fomento de una sociedad más justa al fomentar la diversidad y la participación.

- La consolidación y promoción de las diferencias, profundizando en la cultura propia del entorno y permitiendo que el conocimiento de lo cercano permita una mirada crítica hacia lo lejano.

- El traspaso del individualismo hacia la individualidad potenciado el valor de lo común en una educación de calidad

Si las afirmaciones anteriores son ciertas, si la escuela rural es un espacio propicio para generar respuestas educativas innovadoras y de calidad, cabe preguntarnos ¿qué necesita para que se convierta en realidad?

\section{La Visibilidad como Elemento Potenciador del Cambio}

Tal y como hemos indicado anteriormente la política educativa ha llevado a un cierto desprestigio a la escuela rural por lo que es necesario un impulso para el cambio atendiendo a lo que la propia comunidad educativa quiere, y no sólo a lo que interesa desde el punto de vista político. De este modo la escuela y la educación se convertirán en un medio idóneo de transformación y de desarrollo, ya que la formación constituye un eje fundamental para la transformación del entorno.

La escuela rural precisa asumir un papel activo, protagonizado por los diferentes agentes y actores educativos, que sirva para superar el determinismo y el fatalismo social y que le otorgue un modelo organizativo y pedagógico adaptado a sus peculiares características. No obstante, este papel no podrá ser asumido sin la existencia de políticas activas que tengan en cuenta que el respeto a los diferentes valores e intereses es uno de los pilares del desarrollo sostenible. La apuesta por una educación de calidad en el medio rural es una importante ocasión para desarrollar una innovación pedagógica cuyo eje de atención es la atención a la diversidad y la inmersión de la institución escolar en su medio social.

La diversidad de realidades sociales, políticas, educativas y económicas que contextualizan a la escuela rural precisan de rigurosos 
procesos de descripción e investigación que permitan, primero hacerlas visibles para posteriormente plantear nuevos modelos de escuela rural que sustenten mejoras sociales, educativas y económicas; en definitiva construir conocimiento científico (Boix, 2011). La escuela rural tiene que hacerse ver y por ello es necesario hablar de investigación porque sólo con argumentos rigurosos lograremos esta visibilidad.

Sin embargo los datos de los que disponemos no son halagüeños. Los esfuerzos por comprender el funcionamiento de los centros educativos en entornos rurales no se ha correspondido con una masa crítica suficiente de investigaciones cuyas aportaciones teóricas o empíricas hayan ayudado a lanzar una política educativa que facilitara el sentido de la educación impartida en las escuelas rurales (Martínez; Bustos, 2011). Sin embargo el interés científico por la escuela rural es creciente y por ello hemos considerado interesante resaltar algunas ideas que están presentes en el trabajo de Bustos (2011) en el que revisa el estado de la investigación sobre escuela, enseñanza y/o educación rural a nivel internacional:

- La producción científica, tanto teórica, como empírica sobre escuela rural tiene escasa relevancia en el ámbito pedagógico.

- El ámbito geográfico fundamental en el que se ha desarrollado es Estados Unidos y América Latina ${ }^{3}$.

- Uno de los temas prioritarios ha sido el del alumnado y la comparación de sus resultados académicos con los de la escuela urbana.

- En el ámbito Europeo uno de los temas estrella ha sido la utilización de TIC y la constitución de redes escolares.

- La importancia que adquiere la investigación-acción para proporcionar una visión realista de lo que realmente ocurre en las escuelas rurales.

Además, el conocimiento riguroso de algunas de las características de la escuela rural tales como la atención a la diversidad, el respeto a los ritmos de aprendizaje de los alumnos, el fomento de la autonomía, la responsabilidad y los hábitos de trabajo (Marland, 2004), así como su vinculación al territorio, podrán convertirla en un modelo para el desarrollo de aquellas competencias docentes dirigidas al logro de aprendizajes significativos (Abós, 2011).

La investigación no sólo hace visible la escuela rural sino que proporciona información, para apoyar su mantenimiento, mejorar la formación docente, destacar sus debilidades y fortalezas para actuar sobre las primeras y resaltar las segundas, así como descubrir aquellos elementos sobre los que desarrollar procesos de innovación dirigidos a una educación de calidad para todos.

Con este sentido se plantea el proyecto de investigación que explicamos a continuación y que se encuadra en la Convocatoria de Ayudas a Proyectos de Investigación Fundamental no orientada del Ministerio

672 Educação \& Realidade, Porto Alegre, v. 40, n. 3, p. 667-684, jul./sept. 2015. 
de Ciencia e Innovación de España de $2009^{4}$ con una duración de 3 años (2010-2012) y que implica el reconocimiento de la importancia del tema. Supone un reto pedagógico que por lo que hemos podido constatar carece de antecedentes científico-pedagógicos relevantes.

\section{Nuestro Proyecto: la eficacia y la calidad en la adquisición de competencias caracterizan a la escuela rural: ies un modelo transferible a otra tipología de escuela?}

El proyecto pretende abrir vía de reflexión y diseñar propuestas sobre la adquisición de competencias que caracterizan a la escuela rural y cómo estas competencias trabajadas en el marco de una metodología participativa-activa, con especial atención a la diversidad cultural y social, puede tener componentes didácticos y organizativos transferibles a otra tipología de escuelas. Para ello se analizan experiencias desarrolladas en diferentes contextos de América Latina y de Europa, en un intento de avanzar en la construcción de saberes sobre la escuela multigrado. En este sentido, una de las primeras características que debemos resaltar es el carácter interuniversitario e internacional que presenta el proyecto, potenciando de este modo el análisis de la diversidad de realidades y contextos que caracterizan a la escuela rural en el momento actual. Además, el planteamiento de la investigación desde las instituciones formadoras puede producir diferentes beneficios en cadena con la divulgación de resultados, la potenciación de un nuevo modelo de docente, la mejora de la práctica educativa, la consecución de una educación de calidad, la formación de ciudadanos identificados con su territorio y con la transformación social y la transformación de la escuela rural en un semillero de proyectos pedagógicos dirigidos a la mejora.

Tabla 1 - Países e Instituciones Participantes

\begin{tabular}{|l|l|}
\hline Países & Instituciones participantes \\
\hline Chile & Universidad de Playa Ancha, Valparaíso \\
\hline España & Universidades de Barcelona y Zaragoza \\
\hline Francia & $\begin{array}{l}\text { Observatoire de l’Ecole Rural, Université de Provence, IUFM Aix- } \\
\text { Marseille }\end{array}$ \\
\hline Portugal & Universidad de Lisboa \\
\hline Uruguay & Universidad de La República \\
\hline
\end{tabular}

En cada uno de los países existe un coordinador responsable de su grupo de investigación, siendo diferente el caso de España, donde hay 
uno por cada una de las Comunidades Autónomas participantes (Andalucía, Aragón y Cataluña).

\section{Punto de Partida}

El proyecto se apoya en la caracterización de la escuela rural en función de dos parámetros básicos:

» Situación geográfica en el medio rural.

» Multigraduación como característica escolar fundamental.

\section{Objetivos}

1. Analizar las competencias adquiridas por los alumnos de las escuelas multigrado chilenas, españolas, francesas, portuguesas y uruguayas.

2. Estudiar y comparar la metodología de trabajo en escuelas rurales chilenas, españolas, francesas, portuguesas y uruguayas.

2.1. Analizar las estrategias didácticas utilizadas en aulas multigrado.

2.2. Valorar la organización del espacio y del tiempo en relación a las estrategias didácticas utilizadas.

2.3. Examinar los materiales didácticos utilizados para dar soporte a las estrategias.

2.4. Peritar qué tipo de evaluación y en qué momentos se utiliza.

2.5. Ponderar el interés, la formación y la situación profesional del maestro rural.

2.6. Explorar el contexto socio-cultural y geográfico de las escuelas y las familias.

2.7. Analizar cuáles de los elementos anteriores pueden ser adaptados a otros contextos.

3. Diseñar una propuesta de componentes didácticos que se puedan transferir a otra tipología de escuelas.

3.1. Revisar el estado de la pedagogía de la diversidad en otra tipología de escuelas.

3.2. Elaborar una propuesta de componentes didácticos que se puedan transferir a otra tipología de escuelas, con el intento de lograr aprendizajes de mayor calidad.

\section{Metodología}

En este apartado es importante tener en cuenta no sólo la metodología para la obtención e interpretación de los datos, es decir la metodología del proyecto en sí, sino también la metodología utilizada para

674 Educação \& Realidade, Porto Alegre, v. 40, n. 3, p. 667-684, jul./sept. 2015. 
el trabajo del equipo dadas las características de heterogeneidad del mismo en cuanto a contextos socioculturales y a planteamientos teóricos derivados de la propia tradición en materia de ciencia pedagógica. A ello debemos unir la distancia geográfica y las diferencias horarias entre Europa y América Latina.

\section{a) Metodología del Trabajo en Equipo}

El trabajo del conjunto del equipo puede definirse en función de dos variables fundamentales, una la del trabajo conjunto y otra la del sistema adoptado por cada uno de los subgrupos nacionales. De este modo el equipo internacional ha trabajado a través de la utilización de la Tecnologías de la Información y la Comunicación (Email, Skype, videoconferencia, plataforma de trabajo virtual) y de sesiones de trabajo presenciales, bien de todo el equipo, bien de los coordinadores. En cuanto al trabajo del equipo español ${ }^{5}$ se ha desarrollado con sesiones de trabajo presencial así como con la utilización del Email y Skype.

\section{b) Metodología de Obtención y Tratamiento de la Información}

La metodología del proyecto es predominantemente cualitativa, aunque no se excluye su complementación con técnicas carácter cuantitativo, tanto para la obtención de información como para su análisis. Además la digitalización de mucha de la información permite no sólo un mejor procesamiento de la misma, sino también el intercambio entre los diferentes investigadores. En cuanto a los instrumentos y técnicas debemos destacar los cuestionarios, las entrevistas en profundidad y la etnografía escolar.

\section{Diseño de la Muestra}

La muestra está destinada a detectar y describir las metodologías de aula más eficaces para el logro de aprendizajes en relación a las competencias adquiridas en grupos heterogéneos, y en este sentido la selección en base a la eficacia constituye el principio de selección más recomendable, al que añadimos el de heterogeneidad. Sin embargo aquí nos encontramos con la primera dificultad, ya que en algunos de los países (Portugal y España) no hemos podido tener acceso a los datos de eficacia en base a los resultados obtenidos por el alumnado en pruebas de carácter nacional, en el caso de Portugal, o autonómico en el caso de España. 
El Modelo de Escuela Rural ¿Es un Modelo Transferible a Otro Tipo de Escuela?

Tabla 2 - Criterios de selección de las muestras

\begin{tabular}{|l|l|l|}
\hline Países & Criterios & Heterogeneidad \\
\hline Chile & Eficacia & $\begin{array}{l}\text { Zesultados pruebas } \\
\text { nacionales }\end{array}$ \\
\hline España & Zonasráficas \\
\hline Francia & $\begin{array}{l}\text { Resultados pruebas } \\
\text { nacionales }\end{array}$ & $\begin{array}{l}\text { Zonas geográficas, tamaño, } \\
\text { heterogeneidad (inmigración) }\end{array}$ \\
\hline Portugal & Zonas geográficas \\
\hline Uruguay & $\begin{array}{l}\text { Resultados pruebas } \\
\text { nacionales }\end{array}$ & $\begin{array}{l}\text { Zonas geográficas, tamaño, } \\
\text { heterogeneidad }\end{array}$ \\
\hline
\end{tabular}

Los datos anteriores nos indican que estamos ante una muestra dirigida, seleccionada en base a propiedades identificadas previamente, a criterios definidos a priori, sin aleatorización o criterios estadísticos.

En cuanto al tamaño de la muestra, en la primera etapa de trabajo de campo se consideró el número de 40 aulas por país como un número que permitía concentrar los recursos de investigación para profundizar en los casos y a la vez proveía de una amplitud razonable de indagación y validación de experiencias y procedimientos, aunque señalando la posibilidad de modificación adecuándose a la realidad de cada uno.

Tabla 3 - Tamaño de la Muestra

\begin{tabular}{|l|l|l|}
\hline \multicolumn{1}{|c|}{ Países } & \multicolumn{1}{c|}{ Premuestra } & \multicolumn{1}{c|}{ Muestra } \\
\hline Chile & 54 & 30 \\
\hline España & 159 & 40 (16 Andalucía, 14 Cataluña y 10 Aragón) \\
\hline Francia & & 36 \\
\hline Portugal & & 25 \\
\hline Uruguay & & 17 \\
\hline Total aulas & & 148 \\
\hline
\end{tabular}

Los datos de la tabla nos indican que sólo en Chile y en España se realizó una premuestra a partir de la cual seleccionar la muestra definitiva.

\section{a) Instrumentos Utilizados: cuestionarios}

En esta primera etapa el instrumento utilizado en todos los territorios ha sido un cuestionario que, aunque con diferentes formatos ${ }^{6}$, se ha centrado en la obtención de la siguiente información:

- Datos descriptivos del contexto de la escuela

- Datos descriptivos del centro y del aula 
- Perfil del docente

- Organización del espacio y del tiempo en el aula

- Características del proceso de enseñanza-aprendizaje

- Estrategias didácticas

○ Recursos y materiales

- Sistemas de evaluación

La información obtenida ha servido para la selección de aquellas aulas en las que, según las contestaciones de los docentes, se utilizan de manera más eficiente aquellos procedimientos necesarios para dar respuesta a las diferencias individuales y estimular el aprendizaje autónomo y colaborativo del alumnado; en las que se utilizan estrategias que facilitan la circulación de saberes en el aula, implican una diversificación de actividades según capacidades e intereses y desarrollan contenidos en torno a temas estructurados de enseñanza participativa.

La cumplimentación de estos cuestionarios por parte de los maestros ha seguido diferentes vías ${ }^{7}$ en cada uno de los países en función de la disponibilidad de tiempo del equipo investigador, así como de la mayor o menor lejanía de las escuelas elegidas.

\section{b) Características de la Muestra}

Dadas las diferentes modalidades organizativas de la escuela rural en cada uno de los países, así como de los criterios de selección de la muestra anteriormente mencionados, la heterogeneidad es una de los elementos a destacar. En este sentido los siguientes datos están organizados en torno a dos parámetros: el primero si las escuelas a las que pertenecen las aulas de la muestra están agrupadas o no, y el segundo los grupos o niveles que contienen, lo que nos indica el mayor o menor nivel de multigraduación.

Tabla 4 - Características de la Muestra

\begin{tabular}{|c|c|c|}
\hline Países & \multicolumn{2}{|c|}{ Agrupamiento } \\
\hline & SI & NO \\
\hline Chile & 29 & 1 \\
\hline España & 36 & 4 \\
\hline Francia & - & 36 \\
\hline Portugal & 25 & - \\
\hline Uruguay & & 17 \\
\hline Total & 90 & 58 \\
\hline
\end{tabular}

\section{Selección del Estudio de Caso}

Tal y como hemos indicado en el apartado de metodología la investigación tiene un marcado carácter cualitativo por lo que la siguiente 
fase consistió en la selección de aquellas aulas y maestros con los que íbamos a realizar las entrevistas en profundidad, aquellas en las que, en base a las contestaciones de los maestros, se observara la presencia de estrategias didácticas activas, participativas y dirigidas a la consecución de aprendizajes significativos con la ayuda de los recursos del entorno. No obstante las peculiares características de los equipos de investigación y su implicación en otros proyectos, ha implicado que en algún caso, como el francés, se hayan realizado entrevistas a todos los docentes que constituían la muestra.

a) Instrumentos Utilizados: entrevistas

La entrevista nace de una ignorancia consciente por parte del entrevistador quien, lejos de suponer que conoce, a través de su comportamiento exterior, el sentido que los individuos dan a sus actos, se compromete a preguntárselo a los interesados, en términos y con la suficiente profundidad para captar toda la riqueza de su significado (Ruiz Olabuénaga, 1996, p. 171).

El objetivo básico de esta técnica ha sido la profundización de la información obtenida en los cuestionarios para poder realizar la selección de aquellas aulas en las que íbamos a realizar la última de las fases del trabajo de campo, a saber la observación participante. A pesar de las diferencias en los cuestionarios utilizados en cada uno de los países, existe una concordancia entre el formato y contenido de aquellos y las preguntas diseñadas para las entrevistas. Un ejemplo de una entrevista española sirve para ilustrar la idea anterior.

Eje del cuestionario: Organización del espacio y del tiempo escolar

Cuestión planteada: "Hemos visto en el cuestionario que en su aula el alumnado está agrupado de diferentes maneras y que los criterios para realizar estos agrupamientos son muy diversos. Nos gustaría que nos hablase sobre esta situación en clase, sobre cómo van modificándose los agrupamientos y por qué".

\section{Tabla 5 - Entrevistas Realizadas}

\begin{tabular}{|c|c|}
\hline Países & Entrevistas \\
\hline Chile & $44^{*}$ \\
\hline España & 15 (5 en cada comunidad $)$ \\
\hline Francia & $38^{* *}$ \\
\hline Portugal & 10 \\
\hline Uruguay & 15 \\
\hline Total & 122 \\
\hline
\end{tabular}

* En Chile el cuestionario incluía preguntas abiertas semejantes a las de las entrevistas, lo que hace que el número sea superior a otros países. ** Se realizaron entrevistas a todos los integrantes de la muestra. 
Las entrevistas fueron realizadas en las propias escuelas, previo acuerdo con los maestros, por los diferentes miembros de los equipos nacionales. Su grabación ha permitido su posterior trascripción, así como la utilización de programas informáticos para el análisis de contenido. La selección de estos últimos se realizó de acuerdo con la disponibilidad existente, así como las ventajas e inconvenientes de cada uno de ellos, teniendo en cuenta que nos encontrábamos ante cuatro lenguas diferentes (catalán, español, francés y portugués y ante planteamientos divergentes sobre lo que debía suponer el citado análisis, hecho este que ha servido para enriquecer los supuestos teóricos de la investigación.

\section{b) Observación Participante}

La observación participante es un dispositivo de investigación cuya característica principal es, según la presentación clásica de la Escuela de Chicago (Interaccionismo simbólico), supone una combinación conjunta en el trabajo de campo de una observación - que implica cierta distancia - y de una participación - que implica una inmersión del observador en la situación investigada. Esta idea suponía que la observación debía ser llevada a cabo por personas que, por un lado tuvieran formación en esta técnica de investigación, y por otro tuvieran conocimiento de los supuestos teóricos y metodológicos del proyecto, así como de las peculiaridades de la práctica docente de la escuela rural. De este modo, la observación fue realizada en algunos casos por miembros de los propios equipos nacionales y en otros por otras personas (doctorandos, becarios...) escogidas para realizar esta tarea.

La selección de las aulas para la realización de la observación se hizo en base a la información de los cuestionarios y de las entrevistas considerando aquellas que pudieran ser “joyitas” en cuanto a la utilización de metodologías activas y participativas en relación con la adquisición de competencias. Además se consensuó un protocolo de acceso a los centros, respetando la especificidad de cada país que establecía los siguientes elementos mínimos:

- Comunicación a la autoridad administrativa

- Solicitud formal de acceso a las instancias correspondientes en cada caso (autoridades regionales o provinciales, inspección, dirección de centros...)

- Proceso de negociación con los maestros en los que se incluyera compromisos, tiempos y espacios, así como las consideraciones éticas del proceso de investigación. 


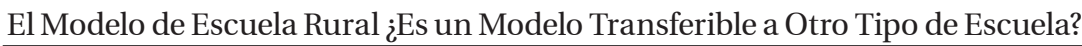

Tabla 6 - Número y Características de las Aulas Observadas

\begin{tabular}{|c|c|c|c|c|}
\hline \multirow[t]{2}{*}{ Países } & \multirow[t]{2}{*}{ Número } & \multicolumn{2}{|c|}{ Agrupamiento } & \multirow[t]{2}{*}{ Niveles/grados } \\
\hline & & Sí & No & \\
\hline Chile & 4 & 4 & - & Clase única $/ 3^{\circ}$ y $4^{\circ}$ \\
\hline España & $\begin{array}{l}3 \text { (Andalucía, Aragón } \\
\text { y Cataluña) }\end{array}$ & 3 & - & $\begin{array}{l}\text { Andalucía: Infantil y } \\
2^{\circ}, 3^{\circ}, 4 \text { y } 6^{\circ} \text { (clase única) } \\
\text { Aragón: } 5^{\circ} \text { y } 6^{\circ} \\
\text { Cataluña: Infantil y } 2^{\circ}, 3^{\circ} \text {, } \\
4^{\circ} \text { y } 5^{\circ} \text { (clase única) }\end{array}$ \\
\hline Francia & $\begin{array}{l}3 \text { (Drôme, Ardèche } \\
\text { y Alpes de Haute } \\
\text { Provence) }\end{array}$ & & 3 & $\begin{array}{l}\text { Clase única (2)/ Tercer } \\
\text { ciclo }\end{array}$ \\
\hline Portugal & 2 & 2 & - & $1^{0}-4^{o}$ \\
\hline Uruguay & $\begin{array}{l}4 \text { (Sur, Centro, Norte } \\
\text { y Oeste) }\end{array}$ & & 4 & $\begin{array}{l}\text { Inicial, } 4^{\circ}, 5^{\circ} y 6^{\circ} \\
1^{\circ}, 3^{o}, 4^{o}, 5^{\circ} y 6^{\circ} \\
1^{\circ}, 3^{\circ}, 4^{o} y 5 \\
\text { Inicial, } 4^{\circ}, 5^{o} y 6^{o} \\
\end{array}$ \\
\hline Total & 16 & 9 & 7 & \\
\hline
\end{tabular}

De acuerdo con los objetivos del proyecto y con los contenidos incluidos en los instrumentos utilizados hasta el momento (cuestionarios y entrevistas), se acordaron las cinco dimensiones que debían ser objeto de evaluación:

- Estrategias didácticas utilizadas por el maestro

- Organización del espacio en el aula

- Organización del tiempo en el aula

- Recursos didácticos utilizados por el maestro y los alumnos

- Evaluación

Al mismo tiempo se decidió que la observación sería realizada de acuerdo al calendario escolar de cada país teniendo en cuenta las diferencias entre Europa y América Latina, con una duración de entre 6 y 8 semanas y de entre 6 y 8 horas semanales, con variación de días de la semana y momentos (mañana/tarde), para garantizar suficiente información sobre la realidad del proceso de enseñanza-aprendizaje observado.

\section{Análisis de Resultados y Situación Actual}

Uno de los elementos más importantes en cualquier proceso de investigación cualitativa es la elección de aquellos instrumentos de análisis de la información que nos permitan adecuarnos más a los objetivos del mismo, así como que mantengan la coherencia con la metodo-

680 Educação \& Realidade, Porto Alegre, v. 40, n. 3, p. 667-684, jul./sept. 2015. 
logía planteada. En este sentido los datos obtenidos hasta el momento son de dos tipos: descriptivos, obtenidos a través de los cuestionarios y que deben ser objeto de un análisis cuantitativo, y de contenido, fruto de las entrevistas en profundidad y de la observación participante, lo que implica la utilización de diferentes técnicas de análisis. En el primero de los casos la explotación de los datos va a ser realizada con el programa SPSS y en el segundo con el programa NUD*IST Vivo que, tal y como señalan Gil y Perera (2001) soporta los procesos de categorización deductiva e inductiva, incluso ambas, lo que posibilita que podamos diseñar a priori un sistema de categorías, en nuestro caso el mismo a lo largo de todo el proceso, así como incorporar otros constructos que surjan de la propia recogida de información. La posibilidad de agrupar y organizar estas categorías va a permitir, además, establecer relaciones y comparaciones.

El análisis cuantitativo ha sido el último que se ha realizado, al considerar que implica menores complicaciones metodológicas, trabajando en los aspectos comunes de los diferentes cuestionarios utilizados para su posterior tratamiento estadístico, y el mayor esfuerzo se ha hecho en la codificación necesaria para realizar el análisis de contenido de las entrevistas y los informes de la observación participante de acuerdo a los objetivos del proyecto. Por poner un ejemplo y dado que el proceso está en fase de finalización, podemos indicar la codificación realizada de la dimensión "Organización del Espacio", teniendo en cuenta que la obtención de los indicadores ha sido tanto deductiva como inductiva a partir de la información aportada por los maestros.

Cuadro 1 - Categoría Organización del Espacio

\begin{tabular}{|c|l|l|}
\hline Categoría & \multicolumn{1}{|c|}{ Subcategorías } & \multicolumn{1}{c|}{ Indicadores } \\
\hline \multirow{4}{*}{$\begin{array}{l}\text { Organización } \\
\text { dePespacio }\end{array}$} & Agrupamientos & $\begin{array}{l}\text { Grados, ciclos, capacidades, } \\
\text { intereses... }\end{array}$ \\
\cline { 2 - 3 } & Criterios & Tamaño del aula, flexibilidad... \\
\cline { 2 - 3 } & Tipos de espacios & $\begin{array}{l}\text { Rincones, talleres, por áreas, por } \\
\text { tipo de actividad... }\end{array}$ \\
\hline
\end{tabular}

Además hemos elaborado un glosario en el que se definen de una manera unívoca los indicadores, ante todo aquellos que puedan ser objeto de diferente acepción en los diferentes territorios, o incluso por los distintos participantes en el proyecto (investigadores y/o maestros).

De manera paralela a este trabajo relacionado con el análisis de datos, se está trabajando en el marco teórico del proyecto dada la necesidad de converger en aquellas ideas que sustentan el mismo y que se refieren fundamentalmente a:

- La concepción de escuela rural y territorio

- La concepción de metodologías activas participativas

- La relación existente entre metodologías activas participativas con el aprendizaje. 


\section{Algunas Valoraciones de la Información Obtenida Hasta el Momento}

Aunque todavía no estamos en condiciones de ofrecer resultados globales del proyecto ${ }^{8}$, sí que podemos realizar algunas valoraciones de la información obtenida hasta el momento:

» La diversidad dentro de la diversidad es una de las características destacables de la escuela rural, lo que puede ayudar al fomento de determinados valores de respeto, colaboración, cooperación...

» En la escuela rural multigrado existen prácticas que son un referente básico para una escuela de calidad, cualquiera que sea el entorno en el que se ubique; entre ellas destacamos :

- Una organización flexible del tiempo y el espacio que posibilita tanto la atención individualizada como la asunción de un modelo cooperativo de trabajo, así como el establecimiento de relaciones interactivas entre alumnos "diferentes".

- Utilización de estrategias didácticas que organizan los contenidos con criterios no exclusivamente disciplinares, lo que permite aprendizajes más significativos.

$\circ \ldots$

\section{Implicaciones de Futuro: crear conciencia y red de investigación}

Aunque en estos momentos no tengamos resultados definitivos que puedan apoyar o refutar las hipótesis de partida, sí podemos poner de manifiesto que la realización del proyecto supone un punto de inflexión en la investigación sobre escuela rural al aproximarnos a puntos de vista convergentes sobre la temática, establecer vínculos y redes entre instituciones y/o personas y hacer visible un tipo de escuela que ha sido y sigue siendo "la hermana pobre", a pesar de contar con grandes posibilidades pedagógicas y sociales en el marco de una sociedad que tiende a la homogeneización y a poner como valor absoluto de calidad los resultados obtenidos en base a criterios uniformes. La igualdad de oportunidades, puesta de manifiesto en una igualdad de resultados debe apoyarse en la supresión de aquellas diferencias que impidan que de verdad podamos ser diferentes.

Recibido en 19 de marzo 2014 Aprobado en 10 de agosto 2014

\section{Notas}

1 EDU2009-13460 (subprograma EDUC) (2010-2012), coordinado por la Dra Roser Boix Tomás.

2 U. Barcelona y U. Zaragoza (España), OER (Francia) , U. Lisboa (Portugal), U. Playa Ancha (Chile) y U. de la República (Uruguay).

682 Educação \& Realidade, Porto Alegre, v. 40, n. 3, p. 667-684, jul./sept. 2015. 
3 En este caso financiada en muchas ocasiones por organismos internacionales (OEI; FAO, Banco Mundial...) con objetivos no estrictamente pedagógicos.

4 Orden de Bases Reguladoras PRE/621/2008 de 7 de marzo (BOE de 8 de marzo) y la Resolución de 26 de diciembre de 2008 (BOE de 31 de diciembre).

5 Del que tenemos toda la información.

6 En el caso de España se ha utilizado una escala Likert.

7 Entrega y recogida personal, email, correo...

8 En la fecha de la publicación del artículo, es decir mayo 2015, el proyecto ya está finalizado.

\section{Referencias}

ABÓS, Pilar. La Escuela en el Medio Rural y su Presencia en los Planes de Estudio de los Grados de Maestro en Educación Infantil y Primaria de las Universidades Españolas. Profesorado. Revista de currículo y formación del profesorado, Granada, v. 15, n. 2, p. 39-52, 2011. Disponible en: <http://www.ugr.es/ recfpro/ rev152ART3.pdf>. Consulta: 10 marzo 2013.

ARÓSTEGUI, José Luis; MARTÍNEZ, Juan B (Coord.). Globalización, Posmodernidad y Educación. La Calidad como Coartada Neoliberal. Madrid: Akal-UNIA, 2008.

BERLANGA, Salvador. Educación en el Medio Rural: análisis, perspectivas y propuestas. Zaragoza: Mira, 2004.

BERNAT, Antonio. Prólogo. In: BERLANGA, Salvador. Educación en el Medio Rural. Análisis, Perspectivas y Propuestas. Zaragoza: Mira, 2003.

BERNAT, Antonio. Escuela rural e ideologías. In: JORNADAS SOBRE EDUCACIÓN EN EL MEDIO RURAL: ENCRUCIJADAS Y RESPUESTAS. Teruel, Universidad de Zaragoza, 2009. Teruel: Universidad de Zaragoza, 2009. DVD.

BOIX, Roser (Coord.). La Escuela Rural: funcionamiento y necesidades. Barcelona: Editorial Praxis, 2004.

BOIX, Roser. Algunas Reflexiones sobre el Valor Pedagógico de lo Rural. 2009. [inédito].

BOIX, Roser. ¿Qué Queda de la Escuela Rural? Algunas Reflexiones sobre la Realidad Pedagógica del Aula Multigrado. Profesorado. Revista de Currículo y Formación del Profesorado, Granada, v. 15, n. 2, p. 13-23, 2011. Disponible en: $<$ http://www.ugr.es/ recfpro/revl52ART1.pdf>. Consulta: 10 marzo 2013.

BUSTOS, Antonio. La Escuela Rural Española Ante un Contexto en Transformación. Revista de Educación, Madrid, n. 350, p. 449-461, 2009. Disponible en: $<$ http://www.revistaeducacion.mec.es/re350/re350_19.pdf>. Consulta: 10 julio 2013.

BUSTOS, Antonio. Investigación y Escuela Rural: ¿irreconciliables? Profesorado. Revista de Currículo y Formación del Profesorado, Granada, v. 15, n. 2, p. 155-170, 2011. Disponible en: <http://www.ugr.es/ recfpro/rev152ART10.pdf>. Consulta: 10 marzo 2013.

FEU, Jordi. La Escuela Rural: apuntes para un debate. Cuadernos de Pedagogía, Barcelona, n. 327, p. 90-94, 2003.

GIL, Javier; PERERA, Victor. Análisis Informatizado de Datos Cualitativos. Sevilla: Kronos, 2001.

Educação \& Realidade, Porto Alegre, v. 40, n. 3, p. 667-684, jul./sept. 2015. 
LORENZO, Manuel; SANTOS, Miguel A. Buscando la Mejora de la Escuela Rural a Través de los Nuevos Entornos Educativos. Revista de Educación, Madrid, n. 335, p. 215-229, 2004.

MARLAND, Paul. Preparing Teachers for Multigrade Classrooms: some questions and answers. University of Southern Queensland, 2004. Disponible en: <http://www.aare.edu.au/93pap/marlp93148.txt>. Consulta: 06 enero 2013.

MARTÍNEZ, Juan B.; BUSTOS, Antonio. Globalización, Nuevas Ruralidades y Escuelas. Profesorado. Revista de Currículo y Formación del Profesorado, Granada, v. 15, n. 2. p. 3-12, 2011. Disponible en: <http://www.ugr.es/ recfpro/rev152ed.pdf>. Consulta: 10 marzo 2013.

MILLER, Ron. A New Culture Needs a New Education. 2006. Disponible en: $<$ http://www.pathsoflearning.net>. Consulta: 10 enero 2013.

POPKEWITZ, Thomas. La Conquista del Alma Infantil. Política de Escolarización y Construcción del Nuevo Docente. Barcelona: Pomares-Corredor, 1998.

ROJAS, José. La Agenda Territorial del Desarrollo Rural en América Latina 2006. Disponible en: <http://www.eumed.net/cursecon/ecolat/la/08/jrl.htm>. Consulta: 10 abril 2013.

RUIZ OLABUENAGA, José I. Metodología de la Investigación Cualitativa. Bilbao: Universidad de Deusto, 1996.

SANTOS, Limber. Aulas Multigrado y Circulación de los Saberes: especificidades didácticas de la escuela rural. Profesorado. Revista de Currículo y Formación del Profesorado, Granada, v. 15, n. 2, p. 72-79, 2011. Disponible en: <http:// www.ugr.es/ recfpro/rev152ART5.pdf>. Consulta: 10 marzo 2013.

SANZ, Alexia. Educación y Territorio. Encrucijadas socioculturales. In: JORNADAS SOBRE EDUCACIÓN EN EL MEDIO RURAL: ENCRUCIJADAS Y RESPUESTAS. Teruel, Universidad de Zaragoza, 2009. Teruel: Universidad de Zaragoza, 2009. DVD.

SEPÚLVEDA, Pilar; GALLARDO, Monsalud. La Escuela Rural en la Sociedad Globalizada: nuevos caminos para una realidad silenciada. Profesorado. Revista de Currículo y Formación del Profesorado, Granada, v. 15, n. 2, p. 141-153, 2011. Disponible en: <http://www.ugr.es/ recfpro/rev152ART9.pdf>. Consulta: 10 marzo 2013.

STAINBACK, Susan; STAINBACK, William. Aulas Inclusivas. Un Nuevo Modo de Enfocar y Unir el Curriculum. Madrid: Narcea, 2007.

Pilar Abós Olivares es profesora titular de Universidad en la Facultad de Ciencias Sociales y Humanas de Teruel, perteneciente a la Universidad de Zaragoza. Directora del Máster Propio en Educación y Desarrollo Rural. Docencia en el Grado de Magisterio de Educación Primaria y en el Máster de Profesorado de Educación Secundaria. Vinculada a los procesos de Gestión de la Calidad de la Universidad de Zaragoza. Investigación vinculada a la escuela rural y a las metodologías didácticas relacionadas con el desarrollo de competencias.

E-mail: pabos@unizar.es 